\title{
9. EIN DIALOGBEGRIFF FÜR DIE MITTELLATEINISGHE LITERATUR
}

In Kapitel 1 wurde die Verwendung des Wortes dialogus als Werkbezeichnung in Titeln, in Aussagen der Autoren und in metaliterarischen Texten untersucht, was zum Ergebnis führte, dass Dialogus im lateinischen Mittelalter mit nur sehr wenigen Ausnahmen auf Prosawerke, die die Personensprache als ausschließliche oder klar dominierende Präsentationsform verwenden, bezogen wird. Diese wenigen Merkmale wurden als Grundlage für die Bildung eines Arbeitskorpus dialogartiger Schriften verwendet. Nach der Untersuchung dieser Schriften ist es nun möglich, einen für die mittellateinische Literatur adäquaten Dialogbegriff zu definieren, der uns die Unterscheidung zwischen Dialogen und ähnlichen Formen erlaubt. Auf dieser Basis wird es auch möglich sein, die Frage zu beantworten, ob die mittellateinischen Dialoge eine einheitliche Textsorte bilden oder ob es mehrere dialogische Textsorten gibt.

\subsection{Die Merkmale eines Dialogs}

Die Untersuchung des Arbeitskorpus zeigt, dass einige Merkmale in der großen Mehrheit der im Arbeitskorpus enthaltenen Schriften vorkommen. Durch diese häufigen Merkmale lässt sich der mittellateinische Dialog beschreiben:

- Die Dialogteilnehmer werden kaum charakterisiert. In einigen Fällen reduzieren sie sich auf einen Namen, so dass die Texte nur durch diese Nennung von Doppeltraktaten oder von Quaestiones et responsiones zu unterscheiden sind. Meistens tragen sie allgemeine Bezeichnungen (Magister, Discipulus, Anima, Ratio, Judaeus u. ä.) und stellen nur einen Typus dar: Homo zeigt Reue und Zerknirschung für seine Sünde, der Discipulus ist lernwillig, der Opponent der Streitgespräche ist widerwillig gegen die Argumente seines Gegners usw. Sie vertreten das Allgemeine und nicht das Singuläre, daher gibt es keine Individuation, auch dann nicht, wenn die Figuren historische Personen darstellen. Der Verfasser tritt zum Beispiel häufig 
als Magister auf, er agiert aber lediglich in dieser Funktion und ist ansonsten nicht als Individuum zu erkennen.

- Die Beziehung zwischen den Figuren (die sich unterschiedlich gestalten kann) bleibt gleich und wird nur so weit gezeichnet, wie es der Darstellung des Inhalts nutzt. Eine gewisse Veränderung ist nur bei den philosophischen Dialogen zu finden, in denen die Figuren zwischen einer Lehrer-Schüler-Beziehung und einer partnerschaftlichen Zusammenarbeit pendeln.

- Die Verfasser der spätmittelalterlichen Dialoge zeigen eine auffällige Bevorzugung der Zwiegespräche. Dieses Merkmal scheint vor allem für den Zeitraum 1200-1400 relevant zu sein: Zum Beispiel haben die biographischen Dialoge des Früh- und Hochmittelalters (bis auf das Werk Wilhelms von Saint Denis) mehrere Gesprächsteilnehmer, wohingegen die moralischen Gespräche nach 1200, die ansonsten große Ähnlichkeit mit den biographischen Dialogen aufweisen, nur zwei Figuren auftreten lassen.

- Der Dialog hat keine oder nur eine sehr reduzierte Rahmenhandlung, die lediglich das Zustandekommen des Gesprächs erklärt und die Figuren einführt. Die Handlung wird während des Gesprächs nicht weiter entwickelt. Die situativen Bezüge sind dementsprechend selten, die meisten Dialoge scheinen außerhalb von Ort und Zeit stattzufinden.

- Die Dialogform kann mehrere Funktionen erfüllen. Sie dient der Gliederung des Inhalts, wobei die Gesprächsanteile einer Figur als Überschriften fungieren. Sie kann auch dazu dienen, unterschiedliche, sogar disparate Inhalte zu integrieren. Durch die Zuschreibung unterschiedlicher Argumente an unterschiedliche Figuren können unvereinbare Positionen klar geschieden werden. Dem Leser kann ferner eine Identifikationsfigur angeboten werden, die seine möglichen Zweifel und Einwände vorwegnimmt oder ihn durch die Äußerung heftiger Emotionen zu einer bestimmten Reaktion (Reue, Wille zur Umkehr, Gottesliebe) hinführt. Seltener kommt eine weitere Funktion zum Tragen, die von Konrad von Hirsau und in den Accessus zu Ciceros De amicitia und De senectute beobachtet wird: der Auftritt eines autoritativen Sprechers, um den Inhalten Nachdruck zu verleihen ${ }^{1}$.

${ }^{1}$ Ermenrich von Ellwangen lässt in seiner Vita Hariolfi den alten Mönch Mahtolf auftreten (siehe oben 91-92), Étienne Gallardon Petrus Cantor (siehe oben 95), der Autor der Visiones Richalmi Abt Richalm (siehe oben 257-259), Albertino Mussato Lovato Lovati (siehe oben 70-71). 
Es gibt keine einfache Korrelation zwischen Typen und Dialogfunktionen. Die Dialogform kann in jedem Typ diese Funktionen erfüllen.

- Dialoge sind inhaltsorientiert: Eine Handlung kommt nicht vor oder wird auf den Rahmen beschränkt; die Dialogform dient mit ihren unterschiedlichen Funktionen der besseren Darstellung der Themen; die Personen sind nur dazu da, Inhalte zu vermitteln oder eine Diskussionsmethode exemplarisch vorzuführen, weshalb ihre Charakterisierung nur dürftig oder nicht vorhanden ist.

- Die Texte sind als Einheit gestaltet. In der Regel bestehen sie aus einem einzigen Gespräch mit gleichbleibenden Teilnehmern, das nur durch Themenwechsel eine Einteilung erfährt.

Diese Eigenschaften definieren den prototypischen mittellateinischen Dialog. Konkrete Dialoge können in dem einen oder anderen Aspekt davon abweichen; solange sie die meisten Merkmale aufweisen, sind sie als Dialoge erkennbar. Einige Beispiele seien hier benannt. Von der mangelnden Charakterisierung der Figuren weicht Petrarcas Secretum $\mathrm{ab}$, in dem Franciscus überwiegend als Individuum mit spezifischen Problemen und Anliegen erscheint. Einige Werke haben mehr als zwei Figuren, zum Beispiel der Trialogus Wyclifs sowie zwei Texte, die einen Disput vor einer Richterfigur inszenieren, der Dialogus des Dondinus Papiensis und De iudiciis viciorum et virtutum des Arnoldus Saxo. Autoren wie Évrard de Trémaugon im Somnium viridarï oder Dondinus Papiensis entwerfen eine Rahmenhandlung für das dargestellte Gespräch, die ausführlicher und sorgfältiger ausgestaltet ist als in den meisten Dialogen. Petrarcas De remediis ist als Sammlung einzelner Dialoge strukturiert; eine gewisse Einheitlichkeit wird nur durch die gleichbleibenden Figuren garantiert.

Wenn eine dialogartige Schrift gleich mehrere dieser Merkmale nicht aufweist, kann sie nicht mehr als Dialog gelten. Einige dieser Werke wurden im Kapitel 8 behandelt. Es wurden auch ganze Textgruppen genannt, die nicht als Dialogtypen gelten können, da die in ihnen erfassten Werke dieser Definition nicht entsprechen. Dazu zählen poetische Textsorten wie die Planctus und die Streitgedichte. Daran ändert die Tatsache nichts, dass einzelne Dialoge diesen Gruppen als Randerscheinung zuzurechnen sind. Auch die dramatischen Allegorien sind eindeutig nicht als Dialoge zu bezeichnen, denn sie weisen geradezu entgegengesetzte Eigenschaften auf: In ihnen ist nicht nur das Thema des Gesprächs wichtig, sondern auch die inszenierte Handlung, die durch die Unterhaltungen der zahlreichen Figuren vorangetrieben wird; 
diese Handlung verlangt einen situativen Rahmen, eine zeitliche und örtliche Einordnung des Geschehens, auf die immer wieder angespielt wird. Die philosophisch-allegorischen Prosimetra sind den dramatischen Allegorien ähnlich gestaltet, nur ein Ausnahmefall (Hildeberts von Lavardin De querimonia) nähert sich der Dialogform an.

Die Streitgespräche über das Heilsgeschehen und die Satansprozesse sind in dieser Beziehung eine geteilte Gruppe. Erstere sind als Dialoge gestaltet, Satansprozesse jedoch nicht. In ihnen treten mehrere Figuren auf, die charakterisiert werden; es gibt eine Handlung und häufige narrative Einschübe. Im letzten und erfolgreichsten Vertreter dieser Gruppe im Spätmittelalter, dem Belial des Jacobus von Theramo, überwiegen sogar deutlich die narrativen Anteile.

Die Schriften aus der Zeit 1200-1400, die unserer Dialogdefinition entsprechen, bilden trotz ihrer formalen Ähnlichkeiten eine heterogene Gruppe. Sie differenzieren sich vor allem unter zwei Gesichtspunkten: den behandelten Themen und der Beziehung zwischen den literarischen Figuren. Unter Berücksichtigung dieser zwei Aspekte lassen sie sich in die vier Typen Lehrdialoge, Streitgespräche, philosophische und selbstbetrachtende Dialoge einteilen. Diese vier großen Typen können wiederum aufgrund verschiedener Merkmale (vor allem inhaltliche, aber auch andersgeartete, wie z. B. die Bindung an einen Entstehungskontext bei den monastischen Lehrdialogen) in Untertypen gegliedert werden. Bei der Beschreibung dieser Typen wurden einzelne Werke berücksichtigt, die Grenzerscheinungen darstellen, da sie nur einzelne Merkmale der Dialogform aufweisen. Als Beispiel sei der Dialogus concupiscentiae et rationis Engelberts von Admont genannt, der ein Zwitter zwischen Dialog und Florileg darstellt: In ihm tritt eigentlich nur eine Figur, Concupiscentia, auf, deren Fragen mit Auflistungen von Zitaten beantwortet werden. Auch Heinrich Seuses Horologium Sapientiae ist eine Einzelerscheinung. Das Werk zeigt zwar Berührungspunkte mit den selbstbetrachtenden Dialogen, doch weicht es auch in wesentlichen Aspekten von der Dialogform ab: Die Personensprache wird immer wieder verlassen oder ist nicht als solche erkennbar, es gibt situative Bezüge und die Beziehung zwischen Discipulus und Sapientia ist immer wieder Änderungen unterworfen. Die größte Originalität zeigen Autoren, die von der lateinischen klerikalen Kultur weniger stark beeinflusst wurden, nämlich drei Laien, Raimundus Lullus, Albertano da Brescia und Inghetto Contardo. Lullus kombiniert sehr frei Elemente aus verschiedenen Traditionen, wie er sie für seine Zwecke gerade braucht. Für die weltlichen und kirchlichen 
Herrscher, die er von der Notwendigkeit der Mission und des Kreuzzugs überzeugen wollte, schrieb er Gespräche mit novellistischen oder dramatischen Elementen. Vor allem die früheren Streitgespräche sind deshalb Randformen des Dialogs. Das Werk des Inghetto Contardo bewegt sich seinerseits am Rand des Typs Streitgespräch; durch die Zahl der Figuren, die Entfaltung einer Handlung, die häufigen narrativen Teile hat es einen fast novellistischen Charakter. Seine Originalität liegt aber vor allem in der spontanen Sprache, die sich immer wieder der Volkssprache annähert. Auch Albertano da Brescia entwickelt eine ausführliche Rahmenhandlung mit novellistischem Charakter.

\subsection{Eine Textsorte Dialog oder dialogische Textsorten?}

Die unter 9.1. beschriebenen Merkmale charakterisieren diejenigen Schriften, die in der mittellateinischen Literatur Dialoge genannt werden können. Dieser Dialogbegriff basiert sowohl auf den direkten oder indirekten Hinweisen, die sich im Mittelalter finden lassen, als auch auf dem heutigen Vergleich der Texte. Nun stellt sich die Frage, ob all die solchermaßen definierten Dialoge eine Textsorte bilden. Mir scheint, dass diese Frage verneint werden muss. Die Dialogmerkmale beziehen sich auf die formale Ausgestaltung der Texte, eine Textsorte wird jedoch auch durch außertextliche Merkmale definiert, wie das Publikum, das sie anspricht, den historischen Kontext, auf den sie sich bezieht, oder das soziale Umfeld, in dem sie entsteht. Diese Aspekte spielen eine wichtige Rolle in der Definition der Typen, die sich durch ein Bündel aus sehr unterschiedlichen Merkmalen beschreiben lassen, weshalb sie als Textsorten betrachtet werden können. Mir scheint angemessen, den Dialog nicht als Textsorte sondern als Form zu definieren: Die Dialogform stellt eine konkrete Ausprägung der Personensprache und somit eine Möglichkeit der literarischen Gestaltung dar, die in verschiedenen Textsorten Anwendung finden kann. Sie ist durch die formalen Merkmale charakterisiert, die oben aufgelistet wurden.

Diese Betrachtung des Dialogs als Dialogform korrespondiert mit der Einstellung der mittellateinischen Autoren, die immer wieder den Dialog als die Präsentationsform eines bestimmten Themas ansprechen. Auch die wenigen metaliterarischen Aussagen versuchen eine Korrelation zwischen Dialog und der Darbietungsform Personensprache, die ihnen jedoch nicht ganz gelingt. 
Wenn wir nun die Dialogform als mögliche Komponente einer Textsorte betrachten, können wir feststellen, dass Lehrdialoge, Streitgespräche, selbstbetrachtende und philosophische Dialoge die Dialogform als konstitutives Merkmal aufweisen, weshalb sie dialogische Textsorten genannt werden können. Allerdings finden sich auch in diesen dialogischen Textsorten einzelne Werke, in denen die Dialogform nicht ausgeprägt ist. Es sind Ausnahmen, die Grenz- und Übergangsformen darstellen. Die dramatischen Allegorien hingegen sind zwar in Personensprache gestaltet, diese weist jedoch andersgeartete Merkmale auf und ist eher als dramatisch zu bezeichnen. Die Streitgespräche über das Heilsgeschehen und die Satansprozesse können, müssen aber nicht, in Dialogform gestaltet sein. Unter ihnen gibt es auch Texte, die eine dramatische Personensprache einsetzen, und andere, die sich nur sekundär der Personensprache bedienen.

In der mittelalterlichen Literatur sind Unterscheidungen nie rigide. Die Autoren kombinieren frei Elemente, die ihnen die Tradition bietet. Die Dialogform lässt sich deshalb nicht auf die dialogischen Textsorten begrenzen, sie kann auch als gestalterische Option in anderen Textsorten vorkommen. In vielen Fällen wurde sie zur gefälligeren Gestaltung formell anspruchsloser Texte (Florilegien, Memoranda, Textbücher) verwendet. Die Form, die durch diese neuen Merkmalskombinationen entsteht, kann so erfolgreich sein, dass eine neue Textsorte entsteht (man denke an die Frage-Antwort-Dialoge, im Grunde Lehrbücher in Dialogform), oder sich auf wenige Fälle beschränken, die wir nun als Randerscheinungen oder Überschneidungen zwischen Textsorten wahrnehmen. Für diese zweite Möglichkeit lassen sich mehrere Beispiele nennen: das dialogisierte Florilegium Engelberts von Admont, die dialogisierten Memoranda von Jean Lefèvre und Perfetto Malatesta, die zwei dialogisierten Planctus, die den Marienklagen zuzurechnen sind, sowie De morte prologus und die Contentio de nobilitate, die sich in mancher Beziehung den Streitgedichten annähern. Die Humanisten des 14. Jahrhunderts greifen für ihre literarisch sorgfältig gestalteten Briefe unter anderem nach der Dialogform. Man müsste der Frage nachgehen, ob die Briefdialoge sich später als eigener Texttyp der Briefliteratur etablierten ${ }^{2}$. Die im Anhang behandelte Predigt des Oglerius von Trino (A7), die einige Marienklagen inspirierte, ist ein Beispiel für

\footnotetext{
${ }^{2}$ Es ist mir nicht gelungen, eine Studie zu diesem Thema zu finden.
} 
die Verwendung der Personensprache in der Predigt. Nun ist die Predigt eine im Spätmittelalter äußerst geläufige Textsorte, von der unzählige Exemplare überliefert sind. Daher könnten sich durchaus noch andere Beispiele für die Ausgestaltung einer Predigt als Dialog finden ${ }^{3}$.

3 Auch in anderen Textsorten dürften Texte in Dialogform noch zu entdecken sein, vgl. zum Beispiel den von Schimmelpfennig, „Fragen an Maria“, edierten Abschnitt aus einer Legenda, der nur aus Fragen besteht, in denen Mariens Teilnahme am Leiden ihres Sohnes geschildert wird. 